\title{
Neurocognitive assessment following whole brain radiation therapy and radiosurgery for patients with cerebral metastases
}

\author{
Susan G R McDuff, ${ }^{1}$ Zachary J Taich, ${ }^{1}$ Joshua D Lawson, ${ }^{2}$ Parag Sanghvi, ${ }^{2}$ \\ Eric T Wong, ${ }^{3}$ Fred G Barker $\|_{1}{ }^{4}$ Fred H Hochberg, ${ }^{4}$ Jay S Loeffler, ${ }^{5}$ Peter C Warnke, ${ }^{6}$ \\ Kevin T Murphy, ${ }^{2}$ Arno J Mundt, ${ }^{2}$ Bob S Carter, ${ }^{1,7}$ Carrie R McDonald, ${ }^{8,9}$ \\ Clark C Chen ${ }^{1,7}$
}

${ }^{1}$ Center for Theoretical and Applied Neuro-Oncology, University of California, La Jolla, California, USA

${ }^{2}$ Department of Radiation Medicine and Applied Sciences, University of California, La Jolla, California, USA

${ }^{3}$ Department of Neurology, Beth Israel Deaconess Medical Center, Boston, Massachusetts, USA

${ }^{4}$ Department of Neurology, Massachusetts General Hospital, Boston, Massachusetts, USA

${ }^{5}$ Department of Radiation Oncology, Massachusetts General Hospital, Boston, Massachusetts, USA

${ }^{6}$ Division of Neurosurgery, University of Chicago Medical Center, Chicago, Illinois, USA ${ }^{7}$ Division of Neurosurgery, University of California, La Jolla, California, USA ${ }^{8}$ Department of Psychiatry, University of California, La Jolla, California, USA ${ }^{9}$ Multimodal Imaging Laboratory, University of California, La Jolla, California, USA

\section{Correspondence to Dr Clark C Chen, \\ Director of Stereotactic and Radiosurgery, Division of Neurosurgery, University of California, San Diego 3855 Health Science Drive \#0987, La Jolla, CA 92093-0987, USA; clarkchen@ucsd.edu SGRM and ZJT contributed equally.}

Received 11 February 2013 Revised 24 April 2013 Accepted 25 April 2013 Published Online First 28 May 2013

To cite: McDuff SGR, Taich ZJ, Lawson JD, et al. J Neurol Neurosurg Psychiatry 2013;84:1384-1391.

\section{ABSTRACT}

The treatment of metastatic brain lesions remains a central challenge in oncology. Because most chemotherapeutic agents do not effectively cross the blood-brain barrier, it is widely accepted that radiation remains the primary modality of treatment. The mode by which radiation should be delivered has, however, become a source of intense controversy in recent years. The controversy involves whether patients with a limited number of brain metastases should undergo whole brain radiation therapy (WBRT) or stereotactic radiosurgery (SRS) delivered only to the radiographically visible tumours. Survival is comparable for patients treated with either modality. Instead, the controversy involves the neurocognitive function (NCF) of radiating cerebrum that appeared radiographically normal relative to effects of the growth from micro-metastatic foci. A fundamental question in this debate involves quantifying the effect of WBRT in patients with cerebral metastasis. To disentangle the effects of WBRT on neurocognition from the effects inherent to the underlying disease, we analysed the results from randomised controlled studies of prophylactic cranial irradiation in oncology patients as well as studies where patients with limited cerebral metastasis were randomised to SRS versus SRS+WBRT. In aggregate, these results suggest deleterious effects of WBRT in select neurocognitive domains. However, there are insufficient data to resolve the controversy of upfront WBRT versus SRS in the management of patients with limited cerebral metastases.

\section{INTRODUCTION}

Metastatic tumours to the cerebrum constitute one of the most common oncological conditions of the adult central nervous system. ${ }^{1}$ Though the exact incidence of cerebral metastases remains unknown, some estimates suggest an annual incidence of 200000 cases per year in the USA. ${ }^{2}$ Between 9\% and $17 \%$ of all cancer patients will develop cerebral metastases during their clinical course. ${ }^{3}{ }^{4}$ The management of patients with cerebral metastasis remains a major challenge in general and neurological oncology.

The incidence of cerebral metastases is projected to increase in the upcoming years for the USA. ${ }^{3}$ There are several reasons that underlie this projection. First, cancer is generally a disease of the elderly, and the average age of the US population is steadily increasing. ${ }^{5}$ As the population of patients at risk for cancer increases, the number of patients at risk for brain metastasis increases. Second, many systemic therapies that are highly effective in treating cancer at its primary site do not penetrate the blood-brain barrier. ${ }^{67}$ For instance, the introduction of trastuzumab, an antibody that interferes with HER2/neu receptor function, has led to significant improvement in the overall survival of patients with breast cancer overexpressing this receptor. ${ }^{8}$ However, as an antibody, trastuzumab penetrates poorly through the blood-brain barrier, and the increased survival in these patients is often associated with elevated risk for cerebral metastasis. ${ }^{3} 69$

The prognosis of patients who develop cerebral metastases remains poor. The median survival is approximately 7 months. ${ }^{10}$ Most studies suggest that cerebral metastases constitute a harbinger of systemic disease progression and that effective chemotherapy remains the key to improving survival in terms of controlling systemic disease progression. ${ }^{11}{ }^{12}$ Despite the poor overall survival, a small subset of patients with metastatic cancer $(1.3 \%-4.2 \%)$ survive beyond 5 years from the time of the diagnosis of cerebral metastasis. ${ }^{11} 13$ While various prognostic scores have been developed for patients of cerebral metastasis, it remains difficult to identify the subset of long-term survivors based on clinical criteria. ${ }^{14}{ }^{15}$ In general, younger age, good performance status, single metastasis, surgical resection, whole brain radiation therapy (WBRT), chemotherapy and lack of extra-cranial disease have been associated with prolonged survival. ${ }^{11} 1516$

In the context of this historically poor survival, the long-term consequences of the various treatments have not been a major focus of oncological research. Major investigative efforts in the past were instead directed toward improving the overall prognosis. ${ }^{15}{ }^{16}$ Emerging data suggest that these efforts are beginning to bear fruition with improved survival in a subset of patients with cerebral metastasis. For instance, the 1-year survival of patients with cerebral metastasis treated between 2005 and 2009 was approximately 34\%. This prognosis compared favourably with those of patients treated during the 1983-1989 period, 
where the 1 -year survival was approximately $15 \% .{ }^{17}$ It is likely that this trend will continue in the future. In this context, there is a renewed interest in assessing the secondary late effects of the various treatment modalities. ${ }^{18}$ Much of this effort has centred on how therapies impact patients' neurocognitive function (NCF).

\section{RADIATION IN THE MANAGEMENT OF CEREBRAL METASTASES}

Therapeutic approaches to cerebral metastases include surgery, radiation therapy, chemotherapy, stereotactic radiosurgery (SRS) or some combination thereof. ${ }^{12} 19$ Of these modalities, radiation remains the core component of current mainstay therapies with other options serving as adjuncts. ${ }^{12} 19$

WBRT is defined as small doses (1.5-2 Gy) of generalised irradiation delivered over 10-30 sessions. The principle behind this form of therapy is that it targets rapidly dividing tumour cells by exploiting the '4Rs of Radiobiology': ${ }^{20}$

- Repair-small doses limit DNA damage allowing normal cells to repair.

- Reoxygenation-areas of hypoxia in the tumour are radioresistant, but small doses allow for increased circulation.

- Redistribution-cells in late S phase are most resistant to radiation, so time must be given between doses to allow for cells to redistribute through the cell cycle.

- Repopulation-irradiated cells repopulate quickly, so treatment must not be excessively delayed.

The efficacy of WBRT in the treatment of cerebral metastases is documented in a large body of literature. ${ }^{21}$ WBRT increases the median survival time by 3-6 months-a therapeutic effect that is unmatched by the other adjunctive modalities, including surgery. $^{22}$ The appropriate combination of WBRT and surgical resection, however, confers significant survival benefits in patients with solitary metastasis. ${ }^{12} 23$

With the development of SRS as a platform for radiation delivery, debate emerged as to whether WBRT is necessary. ${ }^{24}$ SRS delivery techniques use multiple radiation beams to deliver a single large, highly cytotoxic dose of radiation to a discrete tumour target with high precision, thus minimising the radiation dose to the surrounding normal tissue. ${ }^{24} 25$ While many centres favour WBRT in the treatment of patients with cerebral metastases, a growing number of practitioners are opting to treat with focal irradiation.

The issue of controversy generally does not involve survival considerations since the preponderance of data suggests that both modalities yield comparable patient survival. ${ }^{26-28}$ The controversy centres on the issue of cognitive and neurological preservation. Proponents of SRS point to literature suggesting that (1) focal radiation is highly effective in preventing tumour progression in the irradiated volume ${ }^{28}$ and (2) irradiation of normal or near-normal brain tissue increases the risk of cognitive decline in a brain that is already burdened with disease. ${ }^{27}$ These advocates favour a strategy of focal radiation to the radiographically defined lesion followed by close imaging surveillance, with subsequent focal radiation if new lesions arise. ${ }^{27} 28$ On the other hand, proponents of WBRT argue that focal radiation does not address potential micro-metastatic foci that are invisible to conventional imaging. In the absence of radiation treatment, these foci can develop into larger lesions, and it is this disease progression that compromises the patient's neurological and cognitive function. ${ }^{12}$ For WBRT supporters, the risk of disease progression in the brain is thought to outweigh the potential cognitive impairments related to WBRT. ${ }^{12}$ Ultimately, the debate revolves around the tradeoff between preserving the function of cerebrum that is not grossly infiltrated with tumour and the harmful effect of tumour growth from micrometastatic foci.

\section{EFFECTS OF RADIATION ON NEUROCOGNITION}

Fuelling this controversy is an expanding understanding of the importance of neurogenesis in the adult brain as it relates to $\mathrm{NCF}^{29}$ and how this process is compromised by radiation. ${ }^{30}$ It is well accepted that radiation exerts particularly deleterious effects on the neurogenesis that occurs in the developing brain of the paediatric population. There are many studies that document long-term intellectual impairment in paediatric patients, especially younger children, who receive cranial irradiation. ${ }^{31} 32$ As an example, Mulhern et $a l^{33}$ showed a 14-point reduction in IQ for younger children who received WBRT when compared with children irradiated at an older age.

Until recently, it was thought that neurogenesis did not occur in the adult brain. As such, WBRT in adults was assumed to be free of the many deleterious cognitive effects observed in the paediatric population. However, there is growing evidence of actively dividing progenitor cells in regions of the adult brain responsible for memory formation. ${ }^{29}$ There are now compelling data that neural stem cells reside in the human hippocampus and produce dentate granule neurones throughout the entire lifespan. ${ }^{34}$ In experimental models, manipulations that decrease this neurogenesis can impair an animal's performance in hippocampal-dependent behavioural tasks. ${ }^{29} 35$ Conversely, manipulations that increased adult neurogenesis improved cognitive performance in tasks dependent on hippocampal performance. $^{36}$

Importantly, radiation severely compromises the capacity of the hippocampal region to carry out neurogenesis in adult murine models. Monje and Palmer ${ }^{30}$ demonstrated that a single dose of $10 \mathrm{~Gy}$ radiation ablated adult neurogenesis in vivo in murine models. This ablative effect can largely be attributed to changes related to radiation induced inflammatory effects. Accordingly, administration of an anti-inflammatory agent, indomethacin, restored hippocampal neurogenesis in irradiated animals. ${ }^{37}$ Other studies showed that adult mice given $10 \mathrm{~Gy}$ of cranial irradiation to the hippocampus exhibited cognitive impairments in select tasks. ${ }^{35}$ Although these large single fraction experiments may serve as a poor model for clinical practice, other studies have shown similar results using lowered doses of radiation. ${ }^{38}$ Consistent with these findings, postmortem tissue studies showed significantly reduced expression of markers of neurogenesis (eg, doublecortin) in the dentate gyrus of patients who received radiation treatment when compared with age- and sex-matched controls who did not undergo such treatment. ${ }^{39}$

In addition to the compromising effects of irradiation on hippocampal function, there is mounting evidence that irradiation can produce widespread white matter damage by inducing death of the oligodendrocyte progenitor cells and injury to the cerebral vasculature. ${ }^{40}$ Recent studies have shown regions of microscopic white matter changes following irradiation, reflecting demyelination and axonal degradation of the affected tissue. ${ }^{41-43}$ These findings were supported by histological evidence of demyelination, axonal degeneration and necrosis by 48 weeks following irradiation. In aggregate, these studies suggest that irradiation produces white matter damage that may underlie the frontalsubcortical impairments described in patients following irradiation.

\section{NEUROCOGNITIVE DOMAINS AND ASSESSMENT TOOLS}

Because NCF is at the heart of the controversy between upfront WBRT versus and serial SRS, an understanding of the cognitive 
domains thought to be most affected by WBRT is a prerequisite to subsequent discussions. These domains include: global cognitive function, memory, executive functioning, attention and graphomotor speed. ${ }^{44}$ In general, effects of WBRT on hippocampal functioning are likely associated with impairments in learning and memory; the effects that WBRT has on frontal-subcortical white matter are likely associated with impairments in verbal retrieval, executive functioning, attention and processing/motor speed. ${ }^{45}$ Here, we review the tools used to assess the domains of cognitive function that are most relevant in oncological patients.

\section{Global neurocognitive functioning}

The Mini-Mental State Exam (MMSE) is perhaps the most used exam for measuring gross NCF in patients with brain metastases. The test can be administered in minutes and can provide some insight into the overall neurological functioning of the patient. Questions are designed to screen the patient's visuoconstructional and language skills, memory, orientation in time and space, and ability to calculate and sustain attention. The MMSE has high test-retest reliability, and is sensitive and specific to diagnoses of dementia. ${ }^{46}$ Of note, the available data reveal that higher MMSE score is associated with improved survival in patients with brain metastasis. ${ }^{47}$ The major criticism of MMSE relates to its insensitivity to all but the most severe cognitive dysfunction. ${ }^{48}$ For example, Meyers and Wefel $^{49}$ reported that MMSE identified only 26 out of 52 patients classified as abnormal based on more sophisticated neuropsychological batteries as having NCF deficits.

\section{Verbal and non-verbal memory}

Memory impairment is among the most frequently reported cognitive complaints of patients following WBRT. ${ }^{50}$ The Hopkins Verbal Learning Test (HVLT) is a quick, repeatable measure of verbal learning and memory, ${ }^{51}$ and is the most frequent verbal memory measure used in brain tumour clinical trials. Patients are read word lists and are tested on their ability to recall and identify these words. In the HVLT-R, a 20-25 min delayed recall trial is added before the final recognition task. ${ }^{52}$ The HVLT-R has been found to be valid and capable of sensitive and specific discrimination of patients with vascular dementia and Alzheimer's from normal individuals. ${ }^{53}$ The Auditory Verbal Learning Test (AVLT) is a similar measure of verbal learning designed to assess immediate memory, interference and retention of information. ${ }^{54}$

Non-verbal memory is less frequently evaluated in the context of neuro-oncology clinical trials, but the Rey-Osterrieth Complex Figure Test (CFT), which requires the examinee to reproduce a complicated drawing by copying and from memory, is occasionally used to evaluate memory for both visual details and their spatial location. ${ }^{55}$

\section{Executive function}

Executive dysfunction is also commonly reported in patients receiving WBRT treatment and may manifest as difficulties with fluency, set-shifting and planning. The Controlled Oral Word Association (COWA) Test provides a relatively quick test of verbal fluency and is believed to place high demands on executive control processes. ${ }^{56}$ In this test, individuals are given $1 \mathrm{~min}$ to name as many words as possible beginning with predetermine letters. The Trail-Making Test (TMT) is a measure of graphomotor speed and set-shifting ${ }^{57}$ and is also used to measure executive functioning. In Part A, patients are asked to draw sequential connections between 20 circled numbers (from 1 to 20) on a piece of paper. In Part B, both numbers and letters are spread around the sheet, and the patient is asked to alternate between the two (eg, 1, A, 2, B). The executive component of the task is often isolated by subtracting the TMT-A time from the TMT-B time. $^{58}$ This subtraction is performed to remove the effects of psychomotor speed (TMT-A) from those that likely represent increased time due to problem with set-shifting (TMT-B).

\section{Sustained attention and information processing speed}

Although less frequently used in clinical trials, the Paced Auditory Serial Addition Test (PASAT) is a challenging measure of sustained attention and information processing speed. ${ }^{59}$ The subjects are given a number every $3 \mathrm{~s}$ and are asked to add the number they just heard with the number they heard before. This is a challenging task that involves sustained attention, working memory and arithmetic capabilities.

\section{Core assessment of NCF in patients with cerebral metastasis}

For the most part, most NCF measures were designed to assess patients without oncological disease burden. It is not until recently that many of these measures were validated in patients with cerebral metastasis. ${ }^{60}$ Recent efforts have focused on establishing consensus regarding which combination of tools is optimal for assessing NCF in brain tumour clinical trials. ${ }^{48} 61$ An unique challenge in the metastatic population is that the patients are often undergoing concurrent chemotherapy and are battling fatigue related to therapy. In this context, it is sometimes difficult to determine whether an aberrant NCF score is related to general fatigue or anatomic disruption related to radiation. As such, it is critical to select a panel of assay that carefully balances between NCF information acquired with the duration of the testing. In this regard, we favour the panel of tests proposed by Meyers et al. ${ }^{48}$ The author demonstrated that this panel of tests is not terribly time consuming, highly sensitive and minimises training effects from repeated administration. The core measurement tool incorporates assessments of memory using the HVLT, verbal fluency using COWA, visuomotor speed using TMT-A and executive function using TMT-B. ${ }^{48}{ }^{61}$ The core composite battery also includes assessment of symptom severity, ability to complete activities of daily living and quality of life. ${ }^{61}$

\section{ASSESSMENT OF NEUROCOGNITION AFTER WBRT}

Disentangling the direct effects of WBRT on neurocognition from the neurocognitive effects of the underlying disease is a complicated issue. ${ }^{62}$ It is well documented that patients with brain tumours (primary or metastatic) frequently suffer from impaired cognitive function. ${ }^{62-65}$ This impairment is magnified with tumour progression. ${ }^{12}$ Given the further confounding deleterious effects of chemotherapy, seizures and anticonvulsant medication on neurocognition, cognitive status for the brain tumour patients is likely a complex function of the intrinsic effects of the tumour, pertinent neuro-anatomy, patient's psychological state and effect of chemo/radiation as modulated by the patient's inherent sensitivity to these DNA damaging agents. ${ }^{66-71}$ In this context, it is not surprising that the true effect of WBRT on neurocognition remains an area of active controversy. Retrospective or prospective studies have yielded results ranging from those finding that WBRT has no detectable effect on neurocognition to those finding strong correlations between WBRT and life-compromising cognitive decline. ${ }^{72} 73$

Given the complexity of this issue, we do not believe resolution can be easily reached by analysis of the various retrospective series. Here we focus on neurocognitive data derived from 
randomised controlled trials designed to evaluate the effect of WBRT on neurocognition as either primary or secondary outcomes. ${ }^{63}$ 74-76 We selected studies where objective neurocognitive assessment (rather than patient report) was performed at baseline and after WBRT. In an attempt to isolate the intrinsic effect of the brain tumour versus the effect of WBRT, we identified studies that investigated the effect of prophylactic cranial irradiation (PCI). In these studies, all patients underwent a pretreatment MRI to exonerate them from cerebral metastases. We felt that this patient population, while imperfect, gives us the most pristine view of the clinical effect of WBRT on NCF. There have been four randomised controlled trials of PCI in patients with small cell lung cancer (SCLC) that used objective measures to assess neurocognitive outcomes, and one for patients with non-small cell lung cancer (NSCLC).

The first study (Arriagada et al) assessed 294 patients randomised to PCI (24 Gy in eight fractions) or no radiation. ${ }^{76}$ The primary end point was occurrence of brain metastasis, and the secondary end point was neurological complication rate and survival. The authors defined 'higher function' (or NCF) using a non-standardised survey including 'temporo-spatial orientation, memory, judgment (critique of an absurd story), language (spontaneous, dictated sentence, and object names), praxis (drawing, dressing, and ideomotor praxis), and mood status.' No information was provided as to how these assessments were performed. Initial assessment was available for 229 patients, and of those individuals a total of 58 were available at 18 months (14\%), and 35 were available at 30 months (10\%). Cumulative incidence of patients suffering a change in 'higher function' was reported at 2 years. The incidence of such change was comparable between the two groups $30 \%$ of the PCI group and $36 \%$ of the non-PCI group, $\mathrm{p}=0.58)$. While this study is the first published randomised study to examine the effect of PCI on NCF, the instruments used to assess NCF were non-standardised and poorly described. Thus, it is difficult to interpret the results of this study in the context of other studies.

A second study (Gregor et al) randomised 314 SCLC patients to PCI or non-PCI of whom 125 (76 PCI, 49 control) were given a baseline evaluation of cognitive functioning. ${ }^{63}$ Primary end points of the study were cranial relapse and survival, and secondary end points were neurological complication rate and survival. A variety of radiation regimens were used based on the preference of the clinician. The regimen of $30 \mathrm{~Gy}$ in $10 \mathrm{frac}-$ tions was most commonly used, but the range was 8-36 Gy delivered (over the course of 1-18 fractions). Neurocognitive assessments included measures of sustained attention and processing speed (PASAT), non-verbal memory (CFT) and verbal memory (AVLT). Approximately $20 \%$ of the patients remained alive at the 2-year follow-up (25\% in the PCI group and $19 \%$ in the no PCI group). Data were available for 125 patients at baseline and approximately $50 \%$ of the living patients thereafter (59 of 106 patients at 6 months; 32 of 54 patients at 1 year). There were no significant differences in overall survival between the cohorts.

The Gregor et al study is the first study to use standardised NCF measure to assess the effect of PCI in a randomised study. The authors reported no statistically significant difference (at 1 year) in the proportion of patients whose NCF were initially intact but became impaired during follow-up. PCI treated patients were more often compromised on measures of sustained attention and verbal memory relative to the nonirradiated patients, though statistical significance is not reported (table 1). Specifically, 31\% of the PCI group was impaired on the PASAT (compared with $17 \%$ of the non-PCI group), and
$69 \%$ of the PCI group was impaired on the AVLT learning component (compared with $40 \%$ of the non-PCI group). However, $0 \%$ of the PCI group was impaired on the AVLT retention component (compared with $38 \%$ among the non-PCI group). Because the authors did not report statistical values with regard to the NCF comparisons, it is difficult to determine the strength of these trends.

A third study (Wolfson et al) examined neurocognitive effects of PCI on 264 SCLC patients who were randomised to either 25 Gy PCI (in 10 fractions) or 36 Gy PCI (in 18 once-daily or 24 twice-daily fractions). ${ }^{74}$ Neurocognitive outcomes were assessed using measures of fluency, set-shifting and verbal memory (ie, COWAT, TMT and HVLT). The primary outcomes were incidence of chronic neurotoxicity and impact on quality of life. There were no significant differences in overall survival between the cohorts. Approximately 50\% of the patients were alive at 1 year (112 out of 264). Neurocognitive data were collected in $>90 \%$ of the participating patients, at baseline. At 12 months, there was a significant increase in the proportion of cognitive decline in the 36 Gy cohort, as defined as a decrease in performance on at least one cognitive measure in the absence of brain metastases $(p=0.02)$. Approximately $90 \%$ of the patients undergoing 36 Gy PCI suffered cognitive decline as compared with approximately $60 \%$ of the 25 Gy cohort.

A fourth study (Le Pechoux et al) examined NCF in a group of 720 patients who were randomised to receive either 25 or 36 Gy PCI. ${ }^{77}$ The primary end point of their study was the incidence of brain metastases at 2 years. Secondary end points were overall disease-free survival at 2 years, neurological functions and quality of life. Though much of this analysis focused on subjective measures of quality of life and cognitive function, three variables were objectively assessed by a physician, including cognitive functions. The cognitive functions were rated by the physicians based on a scale from 0 to 4 (grade 0 : normal; grade 1: minor loss of memory, reason and/or judgment; grade 2: moderate loss of memory, reason and/or judgment; grade 3: major intellectual impairment; grade 4: complete memory loss/ incapable of rational thought). Of the 720 initial patients, 322 had an initial evaluation with at least one follow-up at 12, 24 or 36 months. There was a trend towards significance with the 36 Gy cohort receiving higher ratings of cognitive impairment than the 25 Gy cohort $(p=0.08)$, especially at 12 and 24 months. While the cognitive measure used by Le Pechoux et $a l^{77}$ is less rigorous and likely more subjective relative to those used by Wolfson et al, ${ }^{74}$ it is notable that the general trend of the Le Pechoux study is in agreement with the results presented by Wolfson et al, suggesting that patients subjected to a 36 Gy WBRT regimen are at a greater risk for cognitive decline at 1 year after treatment.

In addition to the data available for PCI of SCLC, one study recently examined the effect of prophylactic WBRT in patients with NSCLC. ${ }^{75}$ In this study, 356 patients with Stage IIIA/B NSCLC were randomised to PCI (30 Gy over 15 fractions) or observation. The primary end point was overall survival, and secondary end points were disease-free survival, NCF and quality of life. Neurocognitive assessments were performed using the MMSE and HVLT, and functional status was assessed using the Activities of Daily Living Scale. Neurocognitive data were collected in $>90 \%$ of the participating patients. The study found no significant differences in MMSE or the Activities of Daily Living Scale at 1 year between the cohorts. However, there was a significant decline in memory performance (as measured by HVLT immediate recall, $\mathrm{p}=0.03$, and delayed recall, $\mathrm{p}=0.008$ ) among those who received PCI at 1 year. In all, $7 \%$ 
Table 1 Summary of randomised controlled trials examining the effect of PCI on NCF and SRS versus WBRT+SRS

\begin{tabular}{|c|c|c|c|c|c|c|}
\hline Reference & Patients & $\begin{array}{l}\text { Primary end } \\
\text { point }\end{array}$ & Dose & Baseline & Main results & $\begin{array}{l}\text { Statistical } \\
\text { significance }\end{array}$ \\
\hline PCl: Arriagada et al ${ }^{76}$ & $n=294$, SCLC & Survival & 24 Gy & $\begin{array}{l}\text { Baseline for 'higher functions' unpublished. Entire } \\
\text { neuropsychological examination: \#free from any abnormalities } \\
\text { Control: } 44 \text { PCI: } 50\end{array}$ & $\begin{array}{l}\text { \% With cognitive impairment assessed by 'cumulative incidence } \\
\text { of changes in neuropsychological assessment' (higher functions)* } \\
\text { Control: } 36 \% \text { PCl: } 30 \%\end{array}$ & N \\
\hline PCl: Gregor et al ${ }^{63}$ & $n=314$, SCLC & Survival & 8-36 Gy & $\begin{array}{l}\text { PASAT } \\
\text { Control: } 24 \% \text { PCI: } 24 \% \\
\text { CFT } \\
\text { Control: } 41 \% \text { PCI: } 42 \% \\
\text { AVLT learning } \\
\text { Control: } 31 \% \text { PCI: } 38 \% \\
\text { AVLT retention } \\
\text { Control: } 27 \% \text { PCI: } 24 \%\end{array}$ & $\begin{array}{l}\% \text { With cognitive impairment assessed by } \\
\text { PASAT (1 year) } \\
\text { Control: } 17 \% \text { PCl: } 31 \% \\
\text { CFT (1 year) } \\
\text { Control: } 17 \% \text { PCl: } 15 \% \\
\text { AVLT learning (1 year) } \\
\text { Control: } 40 \% \text { PCl: } 69 \% \\
\text { AVLT retention (1 year) } \\
\text { Control: } 38 \% \text { PCl: } 0 \%\end{array}$ & N \\
\hline PCl: Wolfson et al $7^{4}$ & $n=264, S C L C$ & NCF & $\begin{array}{l}25 \text { versus } \\
36 \mathrm{~Gy}\end{array}$ & $\begin{array}{l}\text { Statistically significant differences for the COWAT }(p=0.03 \text { ) and } \\
\text { TMT-A (adjusted } p=0.03 \text { ) at baseline among the three treatment } \\
\text { groups }\end{array}$ & $\begin{array}{l}\text { \% With cognitive impairment as defined by significant decrease in } \\
\text { at least one NCF measure (HVLT, COWAT, TMT-A or TMT-B) } \\
25 \text { Gy: } 62 \% ; 36 \text { Gy: } 85 \%\end{array}$ & Y \\
\hline PCl: Le Pechoux et al ${ }^{77}$ & $n=720, S C L C$ & Survival & $\begin{array}{l}25 \text { versus } \\
36 \mathrm{~Gy}\end{array}$ & $\begin{array}{l}\% \text { Of patients with abnormal QoL-cognitive functioning (scale }<75 \text { ) } \\
25 \text { Gy: } 23 \% ; 36 \text { Gy: } 25 \%\end{array}$ & $\begin{array}{l}\text { \% With cognitive impairment at } 36 \text { months as assessed by a } \\
\text { physician using a scale of } 1-4 \text { at } 2 \text { yearst } \\
25 \text { Gy: } 35 \% ; 36 \text { Gy: } 47 \%\end{array}$ & N \\
\hline PCl: Sun et al ${ }^{75}$ & $n=340, N S C L C$ & Survival & 30 Gy & Unavailable. Baseline used for measurement of decline & $\begin{array}{l}\% \text { With cognitive impairment at } 1 \text { year as assessed by HVLT } \\
\text { Control: } 12 \% \text { PCI: } 41 \%\end{array}$ & Y \\
\hline $\begin{array}{l}\text { SRS versus WBRT+SRS: } \\
\text { Aoyama et } a^{26} 78\end{array}$ & $\begin{array}{l}\mathrm{n}=132, \text { misc. } \\
\text { metastases }\end{array}$ & Survival & $\begin{array}{l}30 \text { Gy } \\
\text { WBRT }\end{array}$ & $\begin{array}{l}\text { Insignificant } \\
\text { SRS: } 27 \text { WBRT+SRS: } 27 \\
\text { p=0.86 }\end{array}$ & $\begin{array}{l}\text { Median MMSE score at } 12 \text { months } \\
\text { SRS: } 28 \text { SRS+WBRT: } 27 \\
\% \text { With cognitive impairment assessed at } 12 \text { months as assessed } \\
\text { by MMSE* } \\
\text { SRS: } 32.5 \% \text { SRS+WBRT: } 48.1 \% \\
\% \text { With cognitive impairment assessed at } 36 \text { months as assessed } \\
\text { by MMSE }{ }^{1} \\
\text { SRS: } 48.1 \% \text { SRS+WBRT: } 85.3 \%\end{array}$ & $\mathrm{~N}$ \\
\hline $\begin{array}{l}\text { SRS versus WBRT+SRS: } \\
\text { Chang et } a^{27}\end{array}$ & $\begin{array}{l}\mathrm{n}=58, \text { misc. } \\
\text { metastases }\end{array}$ & NCF & $\begin{array}{l}30 \text { Gy } \\
\text { WBRT }\end{array}$ & $\begin{array}{l}\text { HVLT (total recall) } \\
\text { SRS: }-0.80(1.53) \\
\text { SRS+WBRT: }-1.12(1.50) \\
\text { HVLT (delayed recall) } \\
\text { SRS: }-0.73(1.31) \\
\text { SRS+WBRT: }-1.32(1.74)\end{array}$ & $\begin{array}{l}\text { \% With cognitive impairment assessed at } 3 \text { months by } \\
\text { HVLT (total recall) } \\
\text { SRS: } 24 \% \text { SRS+WBRT: } 52 \% \\
\text { HVLT (delayed recall) } \\
\text { SRS: } 6 \% \text { SRS+WBRT: } 22 \%\end{array}$ & Y \\
\hline
\end{tabular}

*Assessment described as temporo-spatial orientation, memory, judgment (critique of an absurd story), language (spontaneous, dictated sentence and object names), praxis (drawing, dressing and ideomoter praxis) and mood status. Details of how these assessments were made were absent in the manuscript

tPatients could either be rated as normal or have a grade 1 (minor loss of memory, reason and/or judgment), grade 2 (moderate loss of memory, reason and/or judgment), grade 3 (major intellectual impairment) or grade 4 (complete memory loss/ incapable of rational thought) cognitive impairment.

AVLT, Auditory Verbal Learning Test; CFT, Complex Figure Test; COWAT, Controlled Oral Word Association; HVLT, Hopkins Verbal Learning Test; MMSE, Mini-Mental State Exam; NCF, neurocognitive function; NSCLC, non-small cell lung cancer; PASAT,

Paced Auditory Serial Addition Test; PCI, prophylactic cranial irradiation; QoL, quality of life; SCLC, small cell lung cancer; SRS, stereotactic radiosurgery; TMT, Trail-Making Test; WBRT, whole brain radiation therapy. 
of the patients alive at 1 year suffered significant HVLT decline in immediate recall as compared with $26 \%$ of the patients who underwent PCI. Overall, $5 \%$ of the patients alive at 1 year suffered a significant decline in delayed recall as compared with $32 \%$ of the patients who underwent PCI.

In summary, we identified five studies that randomised patients (all with SCLC or NSCLC) to PCI (table 1). NCF was the secondary end point in all but one of the studies (Wolfson et $a l^{74}$ ). Two of these studies (Arriagada et $a l^{76}$ Le Pechoux et $a l^{77}$ ) did not employ a standardised NCF assessment tool. Two studies used standardised NCF tools to compare PCI treated patients versus patients without PCI. Of these two studies, one study (Sun et al) showed that PCI was associated with significant decline in verbal memory 1 year after PCI $(p=0.008){ }^{75}$ The other study (Gregor et al) showed trends toward worsened decline in attention and verbal memory at 1 year after PCI. ${ }^{63}$

Two studies compared the NCF effect of 36 versus $25 \mathrm{~Gy}$ PCI. NCF was the primary end point in one study ${ }^{74}$ and secondary end point in another study. ${ }^{77}$ The former study (Wolfson et al) used a rigorous NCF scale and showed that 36 Gy PCI was associated with significant verbal memory and executive functioning decline $(\mathrm{p}=0.02)$. The second study (Le Pechoux et al) used a less rigorous NCF scale and demonstrated a trend toward worsened NCF decline in patients treated with 36 Gy PCI $(\mathrm{p}=0.08)$.

\section{COMPARISON OF WBRT AND SRS}

To move toward a resolution of WBRT versus SRS in the treatment of cerebral metastasis, the NCF deficits associated with WBRT need to be weighed against the NCF deficit associated with tumour progression. Here we focus on NCF data derived from two randomised controlled studies designed to evaluate this issue. ${ }^{26} 2778$

In the first study, Aoyama et al randomised 132 patients with one to four cerebral metastases with lesions $<3 \mathrm{~cm}$ to either SRS or SRS+WBRT (30 Gy in 10 fractions). ${ }^{26}$ The primary end point was overall survival, and NCF was a secondary end point. The study showed no significant difference between the therapies in terms of survival (8 months in the SRS group and 7.5 months in the SRS+WBRT group). Salvage therapy was required more frequently for SRS patients due to tumour growth outside of the SRS volume (29 vs 11 patients), but death from neurological cause was comparable between the two groups (19.3\% SRS and $22.8 \%$ WBRT+SRS patients). Of the 44 patients who lived 12 months or longer (19 SRS and 25 WBRT+SRS), MMSE data were available for 28 patients. The median pretreatment and post-treatment MMSE at 1 year was comparable in these two populations.

Interestingly, a bi-phasic difference in MMSE score was noted in a 3 -year follow-up. ${ }^{78}$ In the first 18 months, the MMSE appeared better preserved in the WBRT+SRS group. However, this trend is reversed in the long-term survivors. Of the patients who survived $>36$ months, only $22.5 \%$ of the WBRT + SRS patients exhibited preservation of MMSE (defined as a decrease of MMSE to >26) as compared with $42.6 \%$ of the SRS patients. The data suggest that the patients who underwent SRS +WBRT developed precipitous MMSE decline between 27 and 31 months. It is important to note that the number of patients surviving longer than 36 months was quite small and the finding constituted a trend that failed to reach statistical significance.

Based on these data, Aoyama et al ${ }^{26} 78$ proposed that although the addition of WBRT was effective in preventing the immediate (within 18 months) deterioration of NCF relating to microscopic tumour foci, WBRT was a possible cause of continuous deterioration of MMSE scores in long-term survivors. This hypothesis is consistent with the known delayed neurotoxicity associated with WBRT, which may occur 6 months to several years after treatment. ${ }^{79}$

In a second study, Chang et al randomised 58 patients to SRS and SRS+WBRT (30 Gy in 12 fractions). ${ }^{27}$ In contrast to the Aoyama et al study, the primary end point of this study was NCF, and rather than just using the fairly rudimentary MMSE, NCF was assessed using HVLT-R, WAIS-III, TMT-Part A and COWA. The trial was stopped early due to the observation that patients assigned to the WBRT+SRS group were significantly more likely to exhibit a decline in NCF than the SRS-alone group at 4 months as gauged by HVLT-R. At the termination of this study, $89 \%$ of the WBRT+SRS patients had died as compared with $67 \%$ of the SRS patients $(p>0.05)$. As in the Aoyama et al study, salvage therapy was required more frequently for SRS patients due to tumour growth outside of the SRS volume (29 vs 11 patients), but death from neurological cause was comparable between the two groups (26\% SRS and $25 \%$ WBRT+SRS patients). At 4 months, $52 \%$ of the WBRT + SRS patients suffered significant declines in immediate verbal recall compared with $24 \%$ of SRS patients.

There are several limitations to this study. First, the participants were not stratified to groups based on cognitive performance (and there was a trend toward worse baseline cognition on TMT-Part A in the WBRT+SRS group); nevertheless, all parameters of the HVLT-R were comparable between the two groups. Second, the study lacked follow-up beyond 4 months. Armstrong et $a l^{80}$ studied 20 patients who underwent partial radiation therapy for primary brain tumours with a panel of NCF tests, and showed that patients underwent a clinically significant decline and subsequent rebound in verbal retrieval in the first year (as measured by the AVLT). There is therefore reason to believe that the 4-month end point is premature for understanding the full trajectory of NCF change following WBRT. A third key issue with the Chang et al study is that patients receiving WBRT +SRS had a significantly shorter median survival than the SRS group, suggesting patient selection bias may offer an alternate explanation for the observed effect. ${ }^{81}$

\section{CONCLUSIONS AND FUTURE PERSPECTIVES}

Given the heterogeneity of study design and patient population, meaningful synthesis of the data is difficult. The overall trend seems to suggest that WBRT is associated with deleterious effects on NCF, particularly in the domains of verbal memory, executive functioning and processing speed. This effect appeared particularly evident for the long-term survivors. The observation that higher dose regimen ( 36 vs $25 \mathrm{~Gy}$ ) is associated with worsened NCF, despite having comparable dose-fractions, and in fact higher per fraction radiation for the lower dose regimen (1.5-2 vs 2.5 Gy per fraction), is rather instructive. ${ }^{74}$ It is well appreciated that genetic variations render individuals differentially sensitive to radiation. ${ }^{82}$ The most extreme illustrative case of such phenomenon involves the disruption of DNA repair genes, such as ataxia telangiectasia mutated, where the inactivating mutation renders the patient highly sensitive to radiation. ${ }^{83}$ There are other polymorphisms within the human genome that may similarly moderate radiation sensitivity to a lesser, though still clinically pertinent, extent. ${ }^{67-71}$ In this context, it is possible that the biological effect of $25 \mathrm{~Gy}$ in an individual may equate to that of a higher dose in another. As such and given the dose-dependent neurotoxicity of radiation, judicious consideration with regard to WBRT is warranted. It is 
further worth noting that the effect of radiation in the oncology patient is possibly exacerbated by the known neurocognitive effects of chemotherapy. ${ }^{66}$

At the present time, we feel that there are insufficient data to decisively weigh the relative merits of upfront WBRT versus serial SRS in the management of patients with limited cerebral metastasis. We have provided an exhaustive review of the highest available quality evidence with regard to this question of growing concern: what are the neurocognitive effects related to WBRT and to what extent does SRS provide a rational alternative? Notably, the evidence lends no unambiguous support to the all-too-prevalent thesis that WBRT has negligible effects on NCF. Though this conclusion is necessarily modest, it is important even if it is inconclusive. Given the complexity of the issues reviewed in this article, including patient selection bias and inherent deficits associated with cerebral metastasis, we believe that this issue can only be resolved in a thoughtfully designed, randomised clinical trial, with NCF as a primary end point, and using standardised, rigorous and generally accepted NCF measures. ${ }^{49} 84$ Validated NCF measures in patients with cerebral metastasis, including HVLT, COWA and TMT, warrant consideration as primary end points of such trials. ${ }^{48} 84$

Given the potential deleterious effects of WBRT on NCF, efforts should be made to mitigate such effects in clinical scenarios where WBRT is warranted. Pharmacological agents such as the NMDA receptor antagonist, memantine, are currently undergoing clinical trials for mitigating cognitive dysfunction in patients receiving WBRT. ${ }^{18}$ Efforts to improve the focal tumouricidal effect of radiation by concurrent use of tumour-specific radiosensitising agent, motexafin gadolinium, may afford opportunities to decrease the overall dose of radiation delivered to the cerebrum that is unaffected by metastasis. ${ }^{85}$ The finding that adult neurogenesis occurs in the region of the hippocampus suggests that hippocampal sparing WBRT warrants consideration, especially since the hippocampus is rarely the site of metastatic spread. ${ }^{86} 87$ Finally, an improved understanding of how genetic polymorphisms influence radiation sensitivity will yield opportunities to 'individualise' radiation treatment so as to minimise the risk of NCF decline.

Contributors ZJT, SGRM and CCC performed the systematic review of past studies and authored the initial drafts of the manuscript. ETW, FGB, JSL, FHH, CRM, BSC, JDL, KTM, PCW, PS and AJM provided expert critique and guidance during the revision process.

\section{Competing interests None.}

Funding ZT is supported by an educational grant from the San Diego Gamma Knife Center. CCC is supported by the Doris Duke Charitable Foundation, Sontag Foundation, Burroughs Wellcome Fund, Forbeck Foundation, and Kimmel Foundation

Provenance and peer review Not commissioned; externally peer reviewed.

\section{REFERENCES}

1 Walker AE, Robins M, Weinfeld FD. Epidemiology of Brain-Tumors-the National Survey of Intracranial Neoplasms. Neurology 1985;35:219-26.

2 Simpson JR, Mendenhall WM, Schupak KD, et al. Follow-up and retreatment of brain metastasis. American College of Radiology. ACR Appropriateness Criteria. Radiology 2000;215(Suppl):1129-35.

3 Nayak L, Lee EQ, Wen PY. Epidemiology of brain metastases. Curr Oncol Rep 2012;14:48-54.

4 Nussbaum ES, Djalilian HR, Cho KH, et al. Brain metastases. Histology, multiplicity, surgery, and survival. Cancer 1996;78:1781-8.

5 Schneider EL, Guralnik JM. The aging of America. Impact on health care costs. JAMA 1990;263:2335-40.

6 Langer CJ, Mehta MP. Current management of brain metastases, with a focus on systemic options. J Clin Oncol 2005:23:6207-19.

7 Neuwelt E, Abbott NJ, Abrey L, et al. Strategies to advance translational research into brain barriers. Lancet Neurol 2008:7:84-96.
8 Pegram M, Liao J. Trastuzumab treatment in multiple lines: current data and future directions. Clin Breast Cancer 2012;12:10-18.

9 Burstein HJ, Lieberman G, Slamon DJ, et al. Isolated central nervous system metastases in patients with HER2-overexpressing advanced breast cancer treated with first-line trastuzumab-based therapy. Ann Oncol 2005;16:1772-7.

10 Sperduto PW, Chao ST, Sneed PK, et al. Diagnosis-specific prognostic factors, indexes, and treatment outcomes for patients with newly diagnosed brain metastases: a multi-institutional analysis of 4,259 patients. Int J Radiat Oncol Biol Phys 2010;77:655-61.

11 Hall WA, Djalilian HR, Nussbaum ES, et al. Long-term survival with metastatic cancer to the brain. Med Oncol 2000;17:279-86.

12 Patchell RA. The management of brain metastases. Cancer Treat Rev 2003;29:533-40

13 Altundag K, Bondy ML, Mirza NQ, et al. Clinicopathologic characteristics and prognostic factors in 420 metastatic breast cancer patients with central nervous system metastasis. Cancer 2007;110:2640-7.

14 Nieder C, Nestle U, Motaref B, et al. Prognostic factors in brain metastases: should patients be selected for aggressive treatment according to recursive partitioning analysis (RPA) classes? Int J Radiat Oncol Biol Phys 2000;46:297-302.

15 Sperduto PW, Kased N, Roberge D, et al. Summary report on the graded prognostic assessment: an accurate and facile diagnosis-specific tool to estimate survival for patients with brain metastases. J Clin Oncol 2012;30:419-25.

16 Gaspar L, Scott C, Rotman M, et al. Recursive partitioning analysis (RPA) of prognostic factors in three Radiation Therapy Oncology Group (RTOG) brain metastases trials. Int J Radiat Oncol Biol Phys 1997;37:745-51.

17 Nieder C, Spanne 0, Mehta MP, et al. Presentation, patterns of care, and survival in patients with brain metastases: what has changed in the last 20 years? Cancer 2011;117:2505-12.

18 Gehring K, Sitskoorn MM, Aaronson NK, et al. Interventions for cognitive deficits in adults with brain tumours. Lancet Neurol 2008;7:548-60.

19 Eichler AF, Loeffler JS. Multidisciplinary management of brain metastases. Oncologist 2007:12:884-98.

20 Bernier J, Hall EJ, Giaccia A. Radiation oncology: a century of achievements. Nat Rev Cancer 2004:4:737-47.

21 Posner JB. Management of brain metastases. Rev Neurol (Paris) 1992;148:477-87.

22 Patchell RA, Tibbs PA, Regine WF, et al. Postoperative radiotherapy in the treatment of single metastases to the brain: a randomized trial. JAMA 1998;280:1485-9.

23 Andrews DW, Scott CB, Sperduto PW, et al. Whole brain radiation therapy with or without stereotactic radiosurgery boost for patients with one to three brain metastases: phase III results of the RTOG 9508 randomised trial. Lancet 2004;363:1665-72.

24 Vermeulen SS. Whole brain radiotherapy in the treatment of metastatic brain tumors. Semin Surg Oncol 1998;14:64-9.

25 Peacock KH, Lesser GJ. Current therapeutic approaches in patients with brain metastases. Curr Treat Options Oncol 2006;7:479-89.

26 Aoyama $\mathrm{H}$, Shirato $\mathrm{H}$, Tago $\mathrm{M}$, et al. Stereotactic radiosurgery plus whole-brain radiation therapy vs stereotactic radiosurgery alone for treatment of brain metastases: a randomized controlled trial. JAMA 2006:295:2483-91.

27 Chang EL, Wefel JS, Hess KR, et al. Neurocognition in patients with brain metastases treated with radiosurgery or radiosurgery plus whole-brain irradiation: a randomised controlled trial. Lancet Oncol 2009:10:1037-44.

28 Tsao M, Xu W, Sahgal A. A meta-analysis evaluating stereotactic radiosurgery, whole-brain radiotherapy, or both for patients presenting with a limited number of brain metastases. Cancer 2012;118:2486-93.

29 Shors TJ, Miesegaes G, Beylin A, et al. Neurogenesis in the adult is involved in the formation of trace memories. Nature 2001;410:372-6.

30 Monje ML, Palmer T. Radiation injury and neurogenesis. Curr Opin Neurol 2003;16:129-34

31 Grill J, Renaux VK, Bulteau C, et al. Long-term intellectual outcome in children with posterior fossa tumors according to radiation doses and volumes. Int J Radiat Oncol Biol Phys 1999;45:137-45.

32 Hoppe-Hirsch E, Brunet L, Laroussinie F, et al. Intellectual outcome in children with malignant tumors of the posterior fossa: influence of the field of irradiation and quality of surgery. Childs Nerv Syst 1995;11:340-5; discussion 45-6.

33 Mulhern RK, Hancock J, Fairclough D, et al. Neuropsychological status of children treated for brain tumors: a critical review and integrative analysis. Med Pediatr Oncol 1992:20:181-91.

34 Eriksson PS, Perfilieva E, Bjork-Eriksson T, et al. Neurogenesis in the adult human hippocampus. Nat Med 1998;4:1313-17.

35 Raber J, Rola R, LeFevour A, et al. Radiation-induced cognitive impairments are associated with changes in indicators of hippocampal neurogenesis. Radiat Res 2004; 162:39-47.

36 Dong $\mathrm{S}$, Zeng $\mathrm{Q}$, Mitchell ES, et al. Curcumin enhances neurogenesis and cognition in aged rats: implications for transcriptional interactions related to growth and synaptic plasticity. PLoS One 2012;7:e31211.

37 Monje ML, Toda H, Palmer TD. Inflammatory blockade restores adult hippocampal neurogenesis. Science 2003;302:1760-5 
38 Mizumatsu S, Monje ML, Morhardt DR, et al. Extreme sensitivity of adult neurogenesis to low doses of X-irradiation. Cancer Res 2003;63:4021-7.

39 Monje ML, Vogel H, Masek M, et al. Impaired human hippocampal neurogenesis after treatment for central nervous system malignancies. Ann Neurol 2007:62:515-20.

40 Tofilon PJ, Fike JR. The radioresponse of the central nervous system: a dynamic process. Radiat Res 2000;153:357-70.

41 Akiyama K, Tanaka R, Sato M, et al. Cognitive dysfunction and histological findings in adult rats one year after whole brain irradiation. Neurol Med Chir (Tokyo) 2001;41:590-8.

42 Panagiotakos G, Alshamy G, Chan B, et al. Long-term impact of radiation on the stem cell and oligodendrocyte precursors in the brain. PLoS One 2007:2:e588.

43 Wang S, Wu EX, Qiu D, et al. Longitudinal diffusion tensor magnetic resonance imaging study of radiation-induced white matter damage in a rat model. Cancer Res 2009;69:1190-8.

44 Correa DD. Neurocognitive function in brain tumors. Curr Neurol Neurosci Rep 2010;10:232-9.

45 Meyers CA, Geara F, Wong PF, et al. Neurocognitive effects of therapeutic irradiation for base of skull tumors. Int J Radiat Oncol Biol Phys 2000;46:51-5.

46 Tombaugh TN, Mclntyre NJ. The mini-mental state examination: a comprehensive review. J Am Geriatr Soc 1992:40:922-35.

47 Murray KJ, Scott C, Zachariah B, et al. Importance of the mini-mental status examination in the treatment of patients with brain metastases: a report from the Radiation Therapy Oncology Group protocol 91-04. Int J Radiat Oncol Biol Phys 2000:48:59-64.

48 Meyers CA, Rock EP, Fine HA. Refining endpoints in brain tumor clinical trials. J Neurooncol 2012;108:227-30.

49 Meyers CA, Wefel JS. The use of the mini-mental state examination to assess cognitive functioning in cancer trials: no ifs, ands, buts, or sensitivity. J Clin Oncol 2003:21:3557-8

50 Norden $A D$, Wen PY, Kesari S. Brain metastases. Curr Opin Neurol 2005:18:654-61.

51 Brandt J. The hopkins verbal learning test: development of a new memory test with six equivalent forms. Clin Neuropsychol 1991;5:125-42.

52 Benedict RHB, Schretlen D, Groninger L, et al. Hopkins verbal learning test revised: normative data and analysis of inter-form and test-retest reliability. Clin Neuropsychol 1998;12:43-55.

53 Shapiro AM, Benedict RHB, Schretlen D, et al. Construct and concurrent validity of the hopkins verbal learning test-revised. Clin Neuropsychol 1999;13:348-58.

54 Vakil E, Blachstein H. Rey auditory-verbal Learning Test: structure analysis. J Clin Psychol 1993:49:883-90.

55 Bennett-Levy J. Determinants of performance on the rey-osterrieth complex figure test: an analysis, and a new technique for single-case assessment. $\mathrm{Br} J$ Clin Psychol 1984;23(Pt 2):109-19.

56 Bechtoldt HP, Fogel ML, Benton AL. An application of factor-analysis in neuropsychology. Psychol Record 1962;12:147-56.

57 Armitage SG. An analysis of certain psychological tests used for the evaluation of brain injury. Psychol Monogr 1946;60:1-48.

58 Arbuthnott K, Frank J. Trail making test, part B as a measure of executive control: validation using a set-switching paradigm. J Clin Exp Neuropsychol 2000;22:518-28.

59 Gronwall DM. Paced auditory serial-addition task: a measure of recovery from concussion. Percept Mot Skills 1977;44:367-73.

60 Herman MA, Tremont-Lukats I, Meyers CA, et al. Neurocognitive and functional assessment of patients with brain metastases-a pilot study. Am J Clin Oncol-Canc 2003:26:273-79.

61 Meyers CA, Brown PD. Role and relevance of neurocognitive assessment in clinical trials of patients with CNS tumors. J Clin Oncol 2006:24:1305-9.

62 Platta CS, Khuntia D, Mehta MP, et al. Current treatment strategies for brain metastasis and complications from therapeutic techniques: a review of current literature. Am J Clin Oncol 2012:33:398-407.

63 Gregor A, Cull A, Stephens RJ, et al. Prophylactic cranial irradiation is indicated following complete response to induction therapy in small cell lung cancer: results of a multicentre randomised trial. Eur J Cancer 1997;33:1752-58.

64 Grosshans DR, Meyers CA, Allen PK, et al. Neurocognitive function in patients with small cell lung cancer-effect of prophylactic cranial irradiation. Cancer 2008:112:589-95.
65 Komaki R, Meyers CA, Shin DM, et al. Evaluation of cognitive function in patients with limited small-cell lung-cancer prior to and shortly following prophylactic cranial irradiation. Int J Radiat Oncol Biol Phys 1995;33:179-82.

66 Schagen SB, Muller MJ, Boogerd W, et al. Late effects of adjuvant chemotherapy on cognitive function: a follow-up study in breast cancer patients. Ann Oncol 2002;13:1387-97.

67 Millikan RC, Player JS, Decotret AR, et al. Polymorphisms in DNA repair genes, medical exposure to ionizing radiation, and breast cancer risk. Cancer Epidemio Biomarkers Prev 2005; 14:2326-34.

68 Olshan AF, Shaw GM, Millikan RC, et al. Polymorphisms in DNA repair genes as risk factors for spina bifida and orofacial clefts. Am J Med Genet $A$ 2005:135:268-73.

69 Smith TR, Levine EA, Perrier ND, et al. DNA-repair genetic polymorphisms and breast cancer risk. Cancer Epidemiol Biomarkers Prev 2003:12(11 Pt 1):1200-4.

70 Smith TR, Miller MS, Lohman K, et al. Polymorphisms of XRCC1 and XRCC3 genes and susceptibility to breast cancer. Cancer Lett 2003;190:183-90.

71 Wakeford R. The cancer epidemiology of radiation. Oncogene 2004;23:6404-28.

72 Catane R, Schwade JG, Yarr I, et al. Follow-up neurological evaluation in patients with small cell lung-carcinoma treated with prophylactic cranial irradiation and chemotherapy. Int J Radiat Oncol Biol Phys 1981;7:105-09.

73 Johnson BE, Becker B, Goff WB, et al. Neurologic neuropsychologic and computed cranial tomography scan abnormalities in 2-year to 10-year survivors of small-cell lung-cancer. J Clin Oncol 1985;3:1659-67.

74 Wolfson AH, Bae K, Komaki R, et al. Primary analysis of a phase li randomized trial radiation therapy oncology group (Rtog) 0212: impact of different total doses and schedules of prophylactic cranial irradiation on chronic neurotoxicity and quality of life for patients with limited-disease small-cell lung cancer. Int J Radiat Oncol Biol Phys 2011;81:77-84.

75 Sun A, Bae K, Gore EM, et al. Phase III trial of prophylactic cranial irradiation compared with observation in patients with locally advanced non-small-cell lung cancer: neurocognitive and quality-of-life analysis. J Clin Oncol 2011;29:279-86.

76 Arriagada R, Lechevalier T, Borie F, et al. Prophylactic cranial irradiation for patients with small-cell lung-cancer in complete remission. J Natl Cancer Inst 1995:87:183-90.

77 Le Pechoux C, Laplanche A, Faivre-Finn C, et al. Clinical neurological outcome and quality of life among patients with limited small-cell cancer treated with two different doses of prophylactic cranial irradiation in the intergroup phase III trial (PCI99-01, EORTC 22003-08004, RTOG 0212 and IFCT 99-01). Ann Oncol 2011:22:1154-63.

78 Aoyama H, Tago M, Kato N, et al. Neurocognitive function of patients with brain metastasis who received either whole brain radiotherapy plus stereotactic radiosurgery or radiosurgery alone. Int J Radiat Oncol Biol Phys 2007;68:1388-95.

79 Doyle DM, Einhorn LH. Delayed effects of whole brain radiotherapy in germ cell tumor patients with central nervous system metastases. Int I Radiat Oncol Biol Phys 2008:70:1361-4.

80 Armstrong CL, Corn BW, Ruffer JE, et al. Radiotherapeutic effects on brain function: double dissociation of memory systems. Neuropsychiatry Neuropsychol Behav Neurol 2000;13:101-11.

81 Mahmood U, Kwok Y, Regine WF, et al. Whole-brain irradiation for patients with brain metastases: still the standard of care. Lancet Oncol 11:221-2; author reply 23.

82 Hall EJ, Brenner DJ, Worgul B, et al. Genetic susceptibility to radiation. Adv Space Res 2005:35:249-53.

83 Angele S, Romestaing P, Moullan N, et al. ATM haplotypes and cellular response to DNA damage: association with breast cancer risk and clinical radiosensitivity. Cancer Res 2003;63:8717-25.

84 Correa DD, Maron L, Harder $\mathrm{H}$, et al. Cognitive functions in primary central nervous system lymphoma: literature review and assessment guidelines. Ann Oncol 2007;18:1145-51.

85 Mehta MP, Shapiro WR, Phan SC, et al. Motexafin gadolinium combined with prompt whole brain radiotherapy prolongs time to neurologic progression in non-small-cell lung cancer patients with brain metastases: results of a phase III trial. Int J Radiat Oncol Biol Phys 2009;73:1069-76

86 Gondi V, Tome WA, Mehta MP. Why avoid the hippocampus? A comprehensive review. Radiother Oncol 2010;97:370-6.

87 Ghia A, Tome WA, Thomas S, et al. Distribution of brain metastases in relation to the hippocampus: implications for neurocognitive functional preservation. Int $J$ Radiat Oncol Biol Phys 2007:68:971-7. 